\title{
TRANSIENT FREE CONVECTIVE RADIATIVE FLOW BETWEEN VERTICAL PARALLEL PLATES HEATED/COOLED ASYMMETRICALLY WITH HEAT GENERATION AND SLIP CONDITION
}

\author{
P.K. GAUR and R.P. SHARMA \\ Department of Mathematics, JECRC University \\ Jaipur - 303905, INDIA \\ E-mail: pradeep.gaur@jecrcu.edu.in \\ A.K. JHA* \\ Department of Mathematics, J.N. College Madhubani \\ Bihar-847211, INDIA \\ E-mail: itsabhay@rediffmail.com
}

\begin{abstract}
Investigation of an MHD convective flow of viscous, incompressible and electrically conducting fluid through a porous medium bounded by two infinite vertical parallel porous plates is carried out. ForchheimerBrinkman extended Darcy model is assumed to simulate momentum transfer within the porous medium. A magnetic field of uniform strength is applied normal to the plates. The analytical results are evaluated numerically and the presented graphically to discuss in detail the effects of different parameter entering into the problem.
\end{abstract}

Key words: MHD, slip flow, heat generation, thermal radiation.

\section{Introduction}

Flows through fluid saturated media are important in many scientific and engineering problems such as geothermal energy utilization, heat exchangers, nuclear reactor repositories and chemical engineering for filtration processes. Comprehensive reviews of porous media thermal/species convection have been presented by (Kaviany [1]; Pop and Ingham [2]; Ingham and Pop [3]; Vadasz [4]; Vafai [5]; Neild and Bejan [6]). For any application of porous media it is important to account for non-Darcian effects which can be divided into the inertial (Forchheimer) and boundary (Brinkman) effects. A generalized model for the fluid flow through a porous medium of variable porosity was developed to account for inertial effects, and boundary effects. These effects are incorporated by using the general flow model known as the BrinkmanForchheimer-extended Darcy model.

An analysis on the theoretical derivation of the Darcy and Forchheimer models was presented by Irmay [7]. Neale and Nader [8] showed that the Brinkman model considering continuity of the velocity and the shear stress at the interface gives the same results as obtained by using the Darcy model with BeavevsJoseph condition. Kavinay [9] and Nakayama et al. [10] obtained an analytical solution for a forced convection flow problem in a channel filled with a saturated Brinkman- Darcy porous medium. Flow through porous media, considering the Brinkman-Forchheimer extended Darcy model under different physical conditions has been studied by several authors [Cheng and Choudhary [11], Vafai and Kim [12], Nakayama et al. [13], Kladias and Prasad [14], Shenoy [15], Vafai and Kim [16], Whitakar [17], Nield et al. [18],

\footnotetext{
* To whom correspondence should be addressed
} 
Kuznetsov and Austria [19], Nakayama [20], Leong and Jin [21], Singh and Takhar [22], Singh et al. [23], Pal and Mondal [24]].

In recent years, considerable attention has been paid to the analysis of an MHD boundary layer flow and heat transfer of a Newtonian fluid from a vertical plate/channel immersed in a porous medium because of its wide spectrum of applications in engineering processes, especially in the enhanced recovery of petroleum resources, plasma studies, drying of porous solids, thermal insulation and MHD generators. An MHD natural convection flow bounded by parallel plates through porous media was investigated by Rapits et al. [25]. Attia and Kotb [26] analyzed a magnetohydrodynamic flow and heat transfer bounded by two parallel plates. Kim [27] investigated an unsteady MHD convective heat transfer from a semi-infinite vertical plate with inconstant suction through porous media. Attia [28] investigated the effects of variation in the physical variables on the MHD steady flow and heat transfer bounded by parallel plates through porous media. Ahmed [29] studied the effect of an MHD unsteady natural convective motion bounded by an infinite vertical porous media.

Radiation effects have important applications in the processes involving high temperatures and space technology. Recently, developments in hypersonic flights, space vehicles, gas turbines, nuclear power plants and gas cooled nuclear reactors have attracted researchers in. Radiative convective flows have important applications in environmental and industrial processes, e.g., space vehicle re-entry, astrophysical flows, evaporation from large open water reservoirs, fossil fuels and combustion. Radiative convective flows under different physical conditions have been studied by several authors [Das et al. [30], Bakier [31], Sanyal and Adhikari [32], Mebine [33], El-Hakim and Rashad [34], Muthucumarswamy and Kulandivel [35], Singh and Kumar [36], Singh and Garg [37]].

The study of MHD fluid flows and heat transfer in the slip flow regime has important applications in engineering, for example, electric transformers, heating elements, transmission lines, refrigeration coils and power generators. An MHD unsteady flow of a polar fluid with variable permeability past an infinite horizontal plate in a slip-flow regime through porous media was presented by Khandelwal et al. [38]. Transient natural convection viscous incompressible flows with inconstant suction from a vertical plate in a slip flow regime were presented by Sharma and Chaudhary [39]. The effects of periodic heat and mass transfer on the unsteady natural stream with a mean velocity over which a velocity exponentially varying with time is superimposed was investigated by Sharma [40]. Choudhary and Jha [41] studied an MHD micropolar fluid flow from a vertical plate with chemical reaction in a slip-flow regime. Singh and Pathak [42] investigated an MHD oscillatory convective flow past a rotating vertical channel with slip conditions, thermal radiation and Hall current through porous media.

\section{Formulation of the problem}

An unsteady convection flow of a viscous fluid bounded by two upright plates through porous media is considered. The coordinate axes $x^{*}$ - and $y^{*}$ are taken - along and perpendicular to one of the channel plate. Let $d$ be the distance between the plates. Since the plates are of infinite extent, thus the flow variables depend only on $y$ and $t$. Fluid characteristics, excluding density in the buoyancy force term, are assumed to be constant. Initially, temperatures of the plates and fluid are same as $T_{m}{ }^{*}$. When $t^{*}>0$ the temperatures of the plates at $y^{*}=0$ and $y^{\prime}=d$ are instantaneously raised to $T_{h}{ }^{*}$ and $T_{c}{ }^{*}\left(T_{h}{ }^{*}>T_{c}{ }^{*}\right)$, such that $T=T_{h}{ }^{*}+\varepsilon\left(T_{h}{ }^{*}-T_{c}{ }^{*}\right) e^{-n t}$ and which are thereafter maintained constant. A time dependent injection/suction velocity $v^{*}=-v_{0}\left(1+\varepsilon e^{-n^{*} t^{*}}\right)$ is applied at the plate $y^{\prime}=d$ and $y^{\prime}=0$ respectively.

Therefore, under such assumptions, equations governing the flow relevant to the problem may be written as 


$$
\begin{aligned}
& \frac{\partial v^{*}}{\partial y^{*}}=0 \\
& \frac{\partial u^{*}}{\partial t^{*}}-v_{0}\left(1+\varepsilon e^{-n^{*} t^{*}}\right) \frac{\partial u^{*}}{\partial y^{*}}=v_{e f f} \frac{\partial^{2} u^{*}}{\partial y^{* 2}}-\frac{v_{f}}{K} u^{*}-\frac{F}{\sqrt{K}} u^{* 2}+g \beta_{f}\left(T^{*}-T_{m}^{*}\right)-\frac{\sigma}{\rho_{f}} B_{0}{ }^{2} u^{*}, \\
& \frac{\partial T^{*}}{\partial t^{*}}-v_{0}\left(1+\varepsilon e^{-n^{*} t^{*}}\right) \frac{\partial T^{*}}{\partial y^{*}}=\frac{k}{\rho_{f} C_{p}} \frac{\partial^{2} T^{*}}{\partial y^{* 2}}-\frac{Q_{0}}{\rho_{f} C_{p}}\left(T^{*}-T_{m}^{*}\right)-\frac{\partial q_{r}^{*}}{\partial y^{*}} \frac{1}{\rho_{f} C_{p}} .
\end{aligned}
$$

The relevant boundary conditions are

$$
\begin{aligned}
& t^{*} \leq 0, \quad u^{*}=0, \quad T^{*}=T_{m}^{*} \quad \text { at } \quad 0 \leq y^{*} \leq d, \\
& u^{*}=L_{1} \frac{\partial u^{*}}{\partial y^{*}}, \quad T^{*}=T_{h}^{*}+\varepsilon\left(T_{h}^{*}-T_{c}^{*}\right) e^{-n^{*} t^{*}} \text { at } y^{*}=0, \\
& t^{*}>0, \quad u^{*}=0, \quad T^{*}=T_{c}^{*} \quad \text { at } y^{*}=d \\
& \text { where } \quad L_{l}^{*}=\left(\frac{2-m_{1}}{m_{1}}\right) L .
\end{aligned}
$$

Rosseland's approximation is used for the radiative heat flux which is given below

$$
q_{r}^{*}=-\left(\frac{4 \sigma^{*}}{3 k^{*}} \frac{\partial T^{* 4}}{\partial y^{*}}\right)
$$

The inertia coefficient term $F$ appearing in the model can be evaluated by the following formula (Alazmi et al. [43]; Ergun [45])

$$
F=\frac{1.75}{\sqrt{175 \varepsilon^{3}}}
$$

The dimensionless quantities are defined as

$$
y=\frac{y^{*}}{d}, \quad t=\mathrm{v}_{f} \frac{t^{*}}{d^{2}}, \quad n=\frac{d^{2} n^{*}}{\mathrm{v}_{f}}, \quad u=\frac{u^{*} \mathrm{v}_{f}}{g \beta_{f} d^{2}\left(T_{d}^{*}-T_{c}^{*}\right)}, \quad \theta=\frac{T^{*}-T_{c}^{*}}{T_{d}^{*}-T_{c}^{*}},
$$

$v_{f}=\frac{\mu_{f}}{\rho_{f}}$ (kinematic viscosity), $\lambda=\frac{v_{e f f}}{v_{f}}$ (kinematic viscosity ratio), $S=\frac{d v_{0}}{v_{f}}$ (suction parameter), 
$\mathrm{M}=\frac{\sigma}{\mathrm{v}_{f} \mu_{f}} d^{2} B_{0}{ }^{2} \quad$ (Hartman number), $Q=\frac{Q_{0} d^{2}}{\mathrm{v}_{f} \rho_{f} C_{p}} \quad$ (heat source/sink parameter), Da $=\frac{K}{d^{2}} \quad$ (Darcy number), $R_{4}=\frac{T_{m}^{*}-T_{c}^{*}}{T_{d}^{*}-T_{c}^{*}}$ (buoyancy force distribution parameter), $\mathrm{Gr}=g \beta d^{3} \frac{\left(T_{d}^{\prime}-T_{c}^{\prime}\right)}{\mathrm{v}_{f}}$ (Grashof number), $\operatorname{Pr}=\frac{\mu_{f} C_{p}}{k}$ (Prandtl number), $N_{R}=\frac{k k^{*}}{4 \sigma^{*} T_{\infty}^{* 3}} \quad$ (radiation parameter), $h=\frac{L_{1} v_{0}}{v} \quad$ (slip flow parameter), $k_{2}=\left(\frac{3 N_{R}+4}{3 N_{R}}\right)($ constant $)$

Now introducing the relation Eqs (2.5) and (2.7), into Eqs (2.2) and (2.3), we get

$$
\begin{aligned}
& \frac{\partial u}{\partial t}-S\left(1+\varepsilon e^{-n t}\right) \frac{\partial u}{\partial y}=\lambda \frac{\partial^{2} u}{\partial y^{2}}-\frac{u}{\mathrm{Da}}-\frac{F \mathrm{Gr}}{\sqrt{\mathrm{Da}}} u^{2}+\theta-R_{4}-M u, \\
& \frac{\partial T}{\partial t}-S\left(1+\varepsilon e^{-n t}\right) \frac{\partial T}{\partial y}=k_{2} \frac{1}{\operatorname{Pr}} \frac{\partial^{2} T}{\partial y^{2}}-Q\left(\theta-R_{4}\right),
\end{aligned}
$$

when $R_{4}<0, T_{h}>T_{c}>T_{m}$ while for $R_{4}>0, T_{m}>T_{h}>T_{c}$ and when $0<R_{4}<1$, the wall temperature $T_{h}$ and $T_{c}$ straddle the fluid temperature $T_{m}$.

The dimensionless boundary conditions are

$$
\begin{aligned}
& u=h \frac{\partial u}{\partial y}, \quad \theta=1+\varepsilon e^{-n t} \quad \text { at } \quad y=0, \\
& u=0, \quad \theta=0 \quad \text { at } \quad y=1 .
\end{aligned}
$$

\section{Solution of the problem}

To solve Eqs (2.8) and (2.9), we assumed $\in<<1$ (Gebhart and Pera [45]; Singh et al. [23]) and the solutions to the equations are as follows

$$
\begin{aligned}
& u(y)=u_{0}(y)+\in u_{l}(y) e^{-n t}, \\
& \theta(y)=\theta_{0}(y)+\in \theta_{l}(y) e^{-n t} .
\end{aligned}
$$

Now using the above Eqs (3.1) and (3.2), in Eqs (2.8) to (2.9), we obtain the subsequent equations

$$
\lambda \frac{d^{2} u_{0}}{d y^{2}}+S \frac{d u_{0}}{d y}-\frac{1}{\mathrm{Da}} u_{0}-M u_{0}=\frac{\mathrm{Gr} F}{\sqrt{\mathrm{Da}}} u_{0}^{2}-\theta_{0}+R_{4},
$$




$$
\begin{aligned}
& \lambda \frac{d^{2} u_{l}}{d y^{2}}+S \frac{d u_{1}}{d y}-\frac{1}{\mathrm{Da}} u_{1}-M u_{1}+n u_{1}=\frac{2 \mathrm{Gr} F}{\sqrt{\mathrm{Da}}} u_{0} u_{1}-\theta_{1}, \\
& k_{2} \frac{d^{2} \theta_{0}}{d y^{2}}+\operatorname{Pr} S \frac{d \theta_{0}}{d y}-\operatorname{Pr} Q \theta_{0}=-\operatorname{Pr} R_{4} Q \\
& k_{2} \frac{d^{2} \theta_{1}}{d y^{2}}+\operatorname{Pr} S \frac{d T_{1}}{d y}-\operatorname{Pr}(Q-n) \theta_{1}=-\operatorname{Pr} S \frac{d \theta_{0}}{d y} .
\end{aligned}
$$

The differential Eqs (3.3) and (3.4), are still coupled, so further we assume $F<<1$ (Chamkha [46]) and the solutions to the equations are as follows

$$
u_{0}(y)=u_{00}(y)+F u_{o l}(y), \quad u_{1}(y)=u_{10}(y)+F u_{11}(y) .
$$

Now using the above Eqs (3.7), in Eqs (3.3) to (3.4), we get the following equations

$$
\begin{aligned}
& \lambda \frac{d^{2} u_{00}}{d y^{2}}+S \frac{d u_{00}}{d y}-E_{1} u_{o 0}=-\theta_{0}+R_{4}, \\
& \lambda \frac{d^{2} u_{01}}{d y^{2}}+S \frac{d u_{01}}{d y}-E_{1} u_{01}=\frac{\mathrm{Gr}}{\sqrt{\mathrm{Da}}} u_{00}^{2}, \\
& \lambda \frac{d^{2} u_{10}}{d y^{2}}+S \frac{d u_{10}}{d y}-E_{2} u_{10}=-\theta_{1}-S u_{00} \\
& \lambda \frac{d^{2} u_{11}}{d y^{2}}+S \frac{d u_{11}}{d y}-E_{2} u_{11}=-S u_{01}^{\prime}+\frac{2 \mathrm{Gr}}{\sqrt{\mathrm{Da}}} u_{00} u_{10} .
\end{aligned}
$$

The corresponding boundary conditions (2.10), reduce to the following form

$$
\begin{aligned}
& u_{00}=h \frac{\partial u_{00}}{\partial y}, \quad u_{01}=h \frac{\partial u_{01}}{\partial y}, \quad u_{10}=h \frac{\partial u_{10}}{\partial y}, \quad u_{11}=h \frac{\partial u_{11}}{\partial y}, \theta_{0}=1, \theta_{1}=1 \text { at } y=0, \\
& u_{00}=0, \quad u_{01}=0, \quad u_{10}=0, \quad u_{11}=0, \theta_{0}=0, \theta_{1}=0 \quad \text { at } \quad y=1
\end{aligned}
$$

The solutions to Eqs (3.5) and (3.6), satisfying boundary conditions (3.12) are

$$
\begin{aligned}
& \theta_{0}=F_{1} e^{n_{1} y}+F_{2} e^{n_{2} y}+R_{4}, \\
& \theta_{1}=F_{3} e^{n_{4} y}+F_{4} e^{n_{5} y}-B_{1} e^{n_{2} y}-B_{2} e^{n_{3} y} .
\end{aligned}
$$

Now using Eqs (3.13) and (3.14), in Eq.(3.2), we get $\theta(y)$. 
The solutions to Eqs (3.8) to (3.11) satisfying the boundary conditions (3.12) are

$$
\begin{aligned}
& u_{00}=F_{5} e^{n_{5} y}+F_{6} e^{n_{6} y}-B_{9} e^{n_{1} y}-B_{10} e^{n_{2} y}, \\
& u_{01}=F_{7} e^{n_{5} y}+F_{8} e^{n_{6} y}+B_{15} e^{2 n_{5} y}+B_{16} e^{2 n_{6} y}+B_{17} e^{2 n_{1} y}+B_{18} e^{2 n_{2} y}+ \\
& +B_{19} e^{n_{9} y}-B_{20} e^{n_{10} y}-B_{21} e^{n_{11} y}-B_{22} e^{n_{12} y}-F_{23} e^{n_{13} y}+B_{24} e^{n_{14} y} \\
& u_{10}=F_{9} e^{n_{7} y}+F_{10} e^{n_{8} y}+e^{n_{1} y}\left(B_{28}+B_{32}\right)+e^{n_{2} y}\left(B_{29}+B_{33}\right)+ \\
& -B_{30} e^{n_{3} y}-B_{31} e^{n_{4} y}-B_{34} e^{n_{5} y}-B_{35} e^{n_{6} y} \\
& u_{11}=F_{11} e^{m_{7} y}+F_{12} e^{m_{8} y}-e^{2 n_{5} y} B_{68}-e^{2 n_{6} y} B_{69}-e^{2 n_{2} y} B_{70}-e^{2 n_{1} y} B_{71}-B_{72} e^{n_{9} y}+B_{73} e^{n_{10} y}+ \\
& +B_{74} e^{n_{11} y}+B_{75} e^{n_{12} y}+B_{76} e^{n_{13} y}-B_{77} e^{n_{14} y}+B_{78} e^{n_{19} y}+B_{79} e^{n_{20} y}-B_{80} e^{n_{21} y}-B_{81} e^{n_{22} y}+ \\
& +B_{82} e^{n_{23} y}+B_{83} e^{n_{24} y}-B_{84} e^{n_{25} y}-B_{85} e^{n_{26} y}-B_{86} e^{n_{27} y}-B_{87} e^{n_{28} y}+B_{88} e^{n_{29} y}+B_{89} e^{n_{30} y}+ \\
& -B_{90} e^{n_{31} y}-B_{91} e^{n_{32} y}+B_{92} e^{n_{33} y}+B_{93} e^{n_{34} y}-B_{94} e^{n_{5} y}-B_{95} e^{n_{6} y} .
\end{aligned}
$$

Now using Eqs (3.15) to (3.18) in Eq.(3.7), we get $u_{0}(y)$ and $u_{l}(y)$ respectively, which finally yields $u(y)$ by Eq.(3.1).

\section{Results and discussion}

In order to study the nature of velocity, temperature, and mass transfer, numerical calculations are carried out for distinct values of $R_{4}, S, \mathrm{Gr}, N_{R}, t$, Mand $Q$ which are listed in figures and the results are reported graphically.

Figures 1 to 3 show the effects of time and the buoyancy force parameter on the fluid velocity. When $R_{4}<0$ from Fig. 1 , it is noticed that near the heated plate $(y=0)$ the velocity gets its maximum value and starts falling towards the cold plate $(y=1)$ due to the negative value of the buoyancy force parameter, the temperature of both plates is greater than the fluid temperature. When $0 \leq R_{4} \leq 1$ it is observed in Fig.2 that near the hot plate $(y=0)$ the fluid velocity gets its maximum value and then drops all over the flow area. The reason is that the hot plate fluid is heated. When $R_{4}>1$ it is observed in Fig.3 that the temperature of the cooled plate is lower than the temperature of the fluid, thus near the cooled plate a reverse flow is occurring. The reason is that at the starting stage, the temperature of both the plates is greater than the fluid temperature. The figures it also show that the velocity enhances as the time increases and a steady state is obtained at $t=1$. Figure 4 presents the influence of time and the suction/injection parameter on the velocity. It is noticed that the velocity diminishes with the growing value of the suction/injection parameter. The reason is that the suction/injection parameter enhances the drag force nearby the channel plates. From the figures it also follows that the velocity enhances as the time increases and the steady state is obtained at $t=1$. Figure 5 illustrates the influence of time and the Grashof number on the velocity. It is found that the Grashof number has the leading effect on accelerating velocities. It is also observed that the velocity enhances as the time increases and the steady state is obtained at $t=1$. The influence of the radiation parameter is shown in Fig.6. It is found that the fluid velocity gets its maximum value nearby the heated plate and then diminishes gradually towards the cooled plate. It is also noticed that the velocity enhances as the time increases and the steady state is obtained at $t=1$. The influence of the Hartmann number on the 
fluid velocity is presented in Fig.7. It is observed that the fluid velocity decelerates with the growing value of the Hartmann number the velocity enhances as the time increases and the steady state is obtained at $t=1$.

Figures 8 and 9 represent the influence of heat source /sink Q on the temperature. It can be noticed that the temperature diminishes with the growing value of the heat sink parameter and a similar trend is seen in the case of the heat source parameter. Figure 10 depicts the effect of the radiation parameter on the temperature. It is observed that the temperature profile drops with the growing value of the radiation parameter. Figures 11 to 13 show the effect of the buoyancy force parameter on the temperature. When $R_{4}<0$, the temperature diminishes with rising values of $R_{4}$. A similar behavior is noticed in the case of $0 \leq R_{4}<1$ and $R_{4}>0$. The reason is that the temperature of the cooled plate is lower than the temperature of the heated plate and the temperature of the fluid is lower than the temperature of both plates.

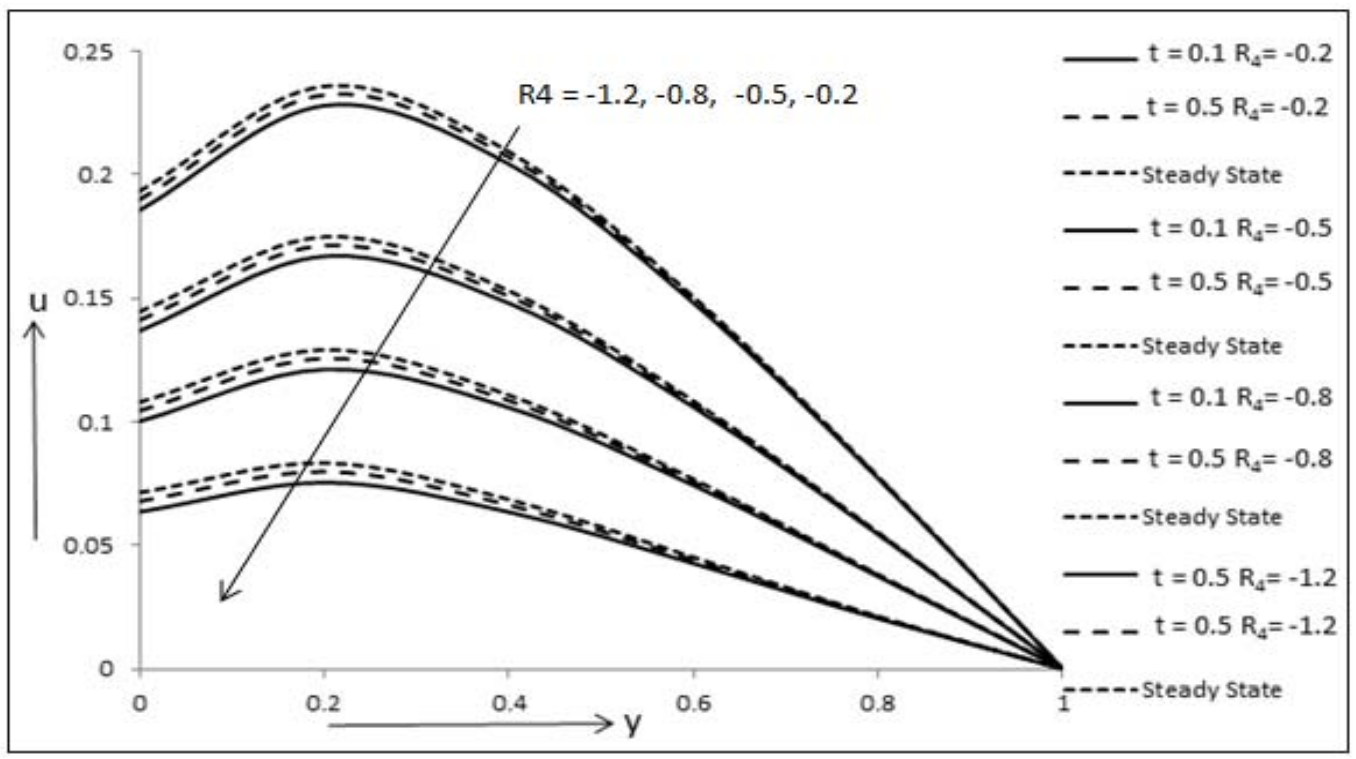

Fig.1. Velocity profiles for various values of the buoyancy force parameter $\left(R_{4}<0\right)$.

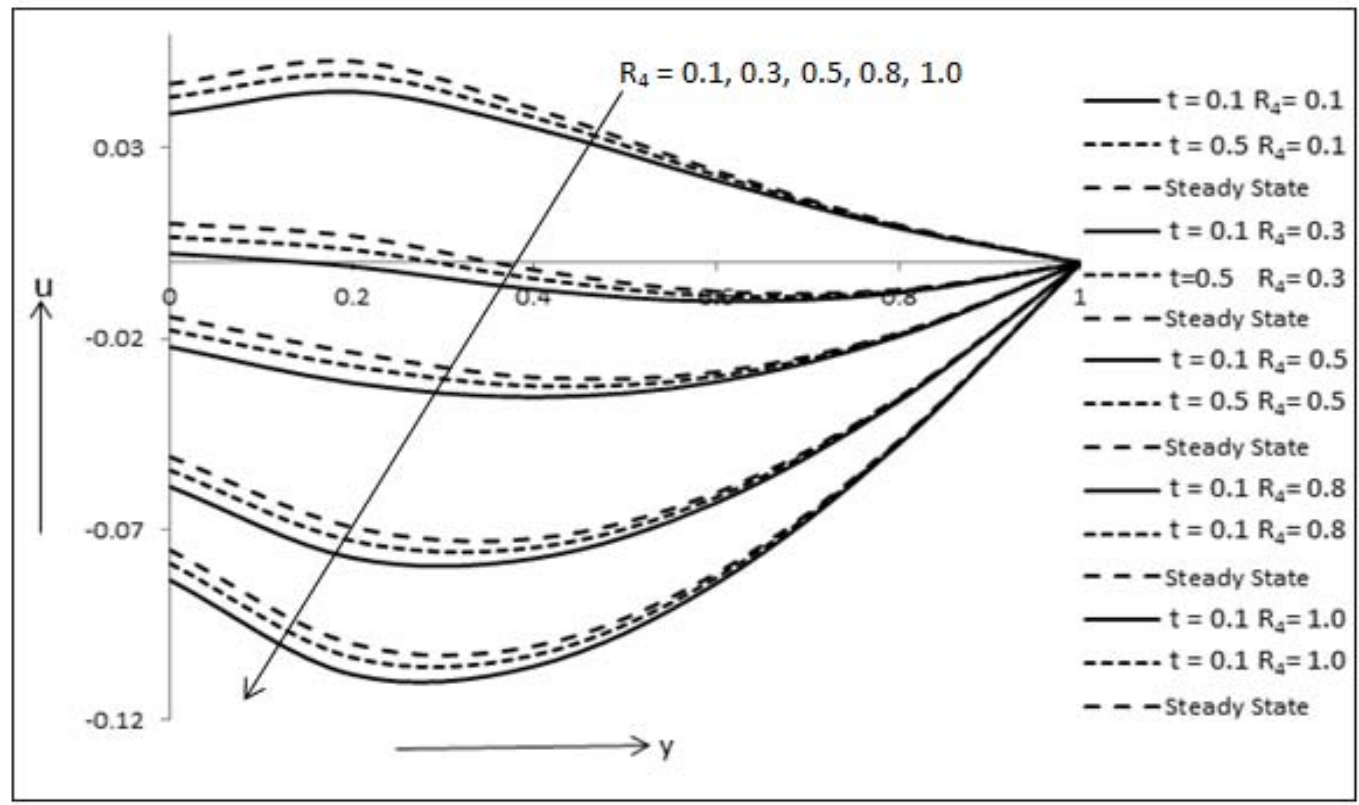

Fig.2. Velocity profiles for various values of the buoyancy force parameter $\left(R_{4}>0\right)$. 


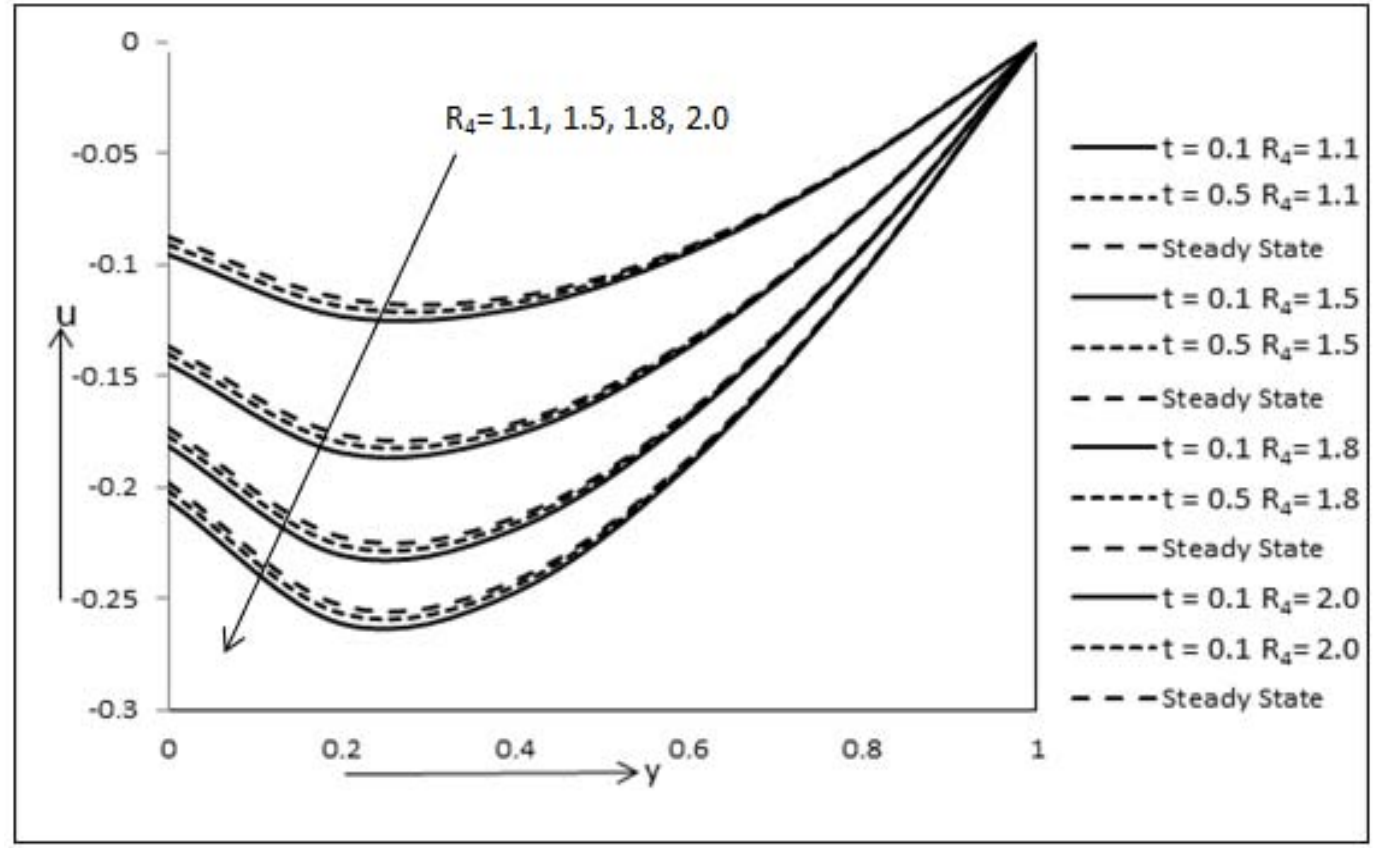

Fig.3. Velocity profiles for various values of the buoyancy force parameter $\left(R_{4}>1\right)$.

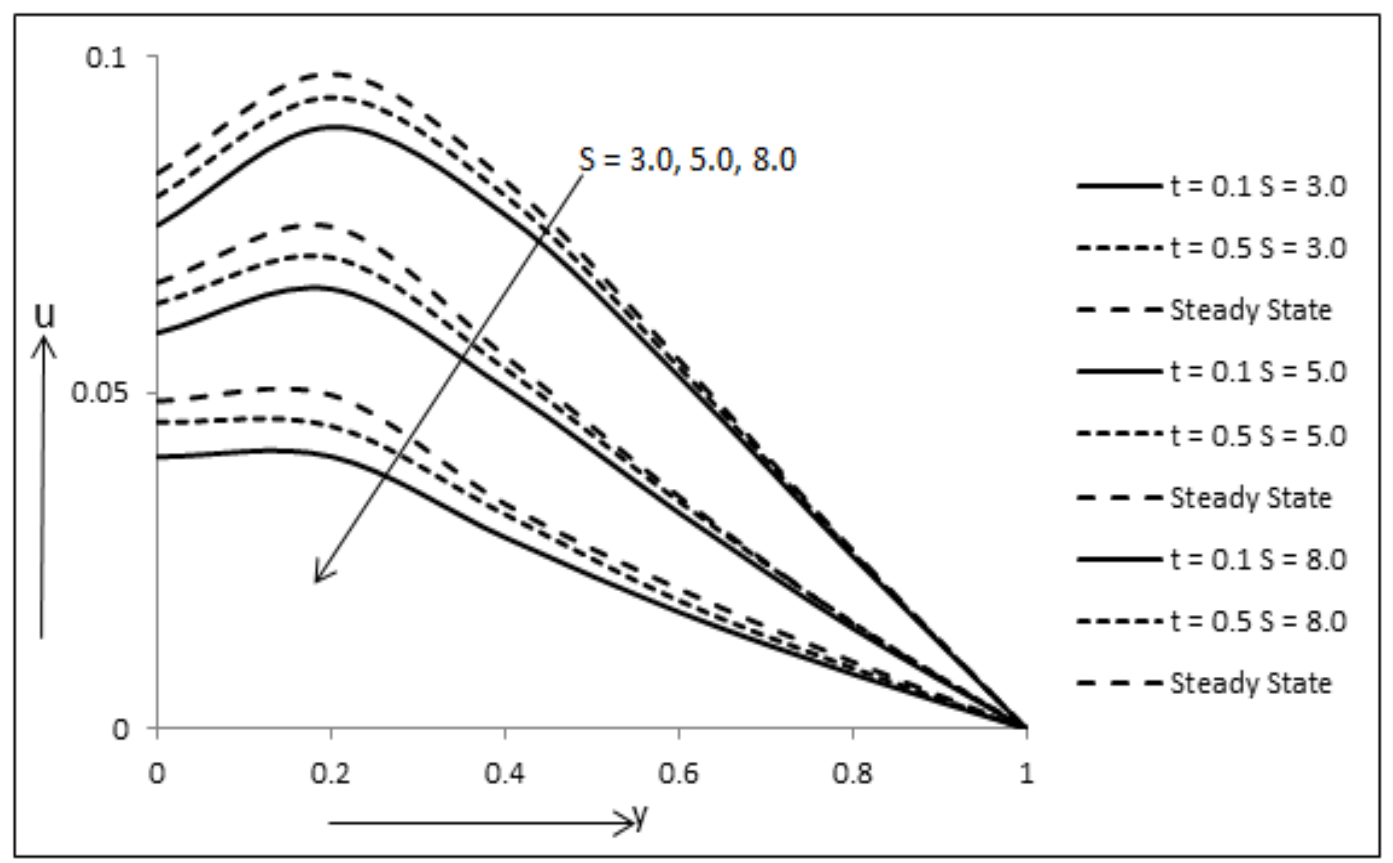

Fig.4. Velocity profiles for various values of the suction/injection parameter. 


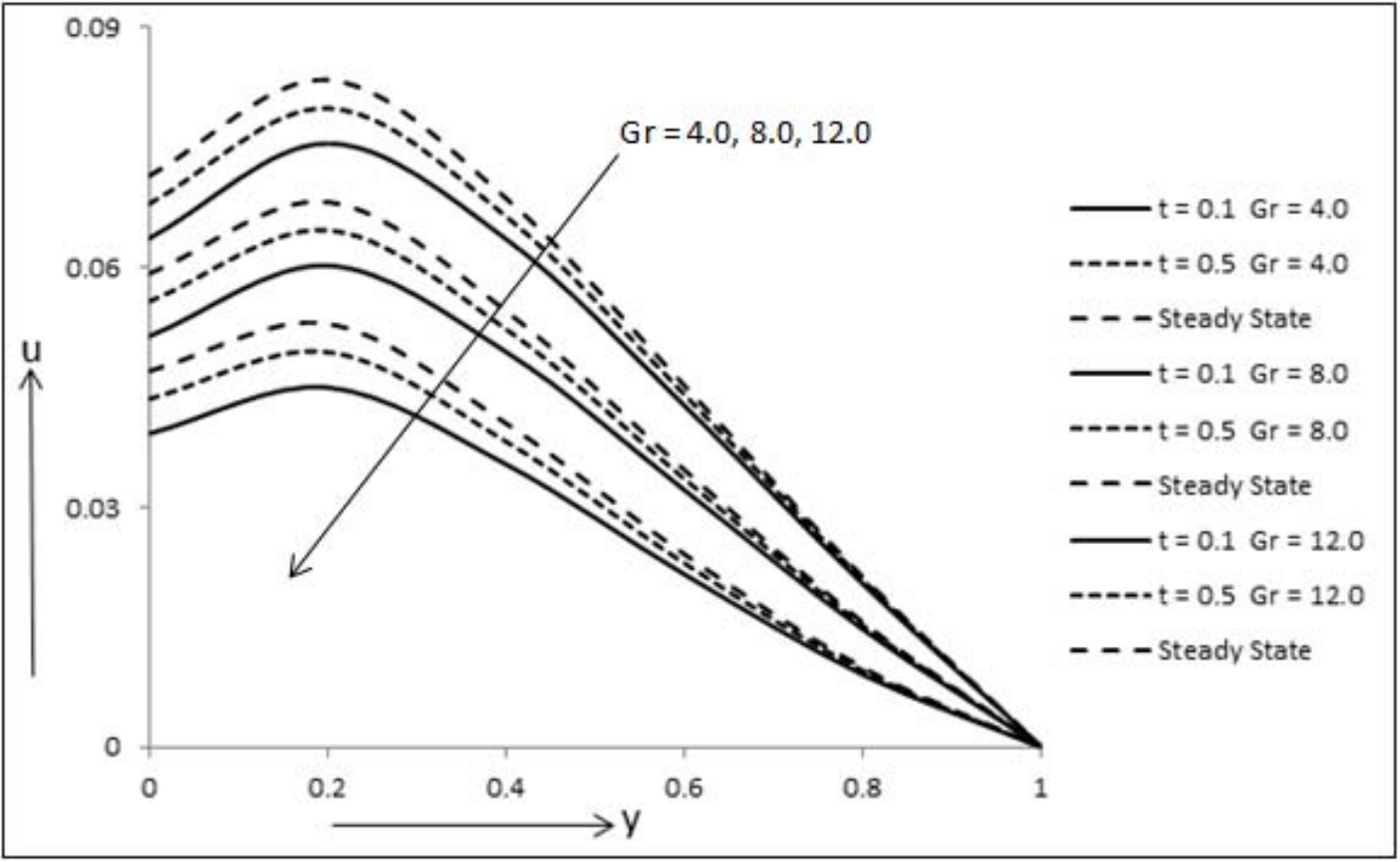

Fig.5. Velocity profiles for various values of the Grashof number.

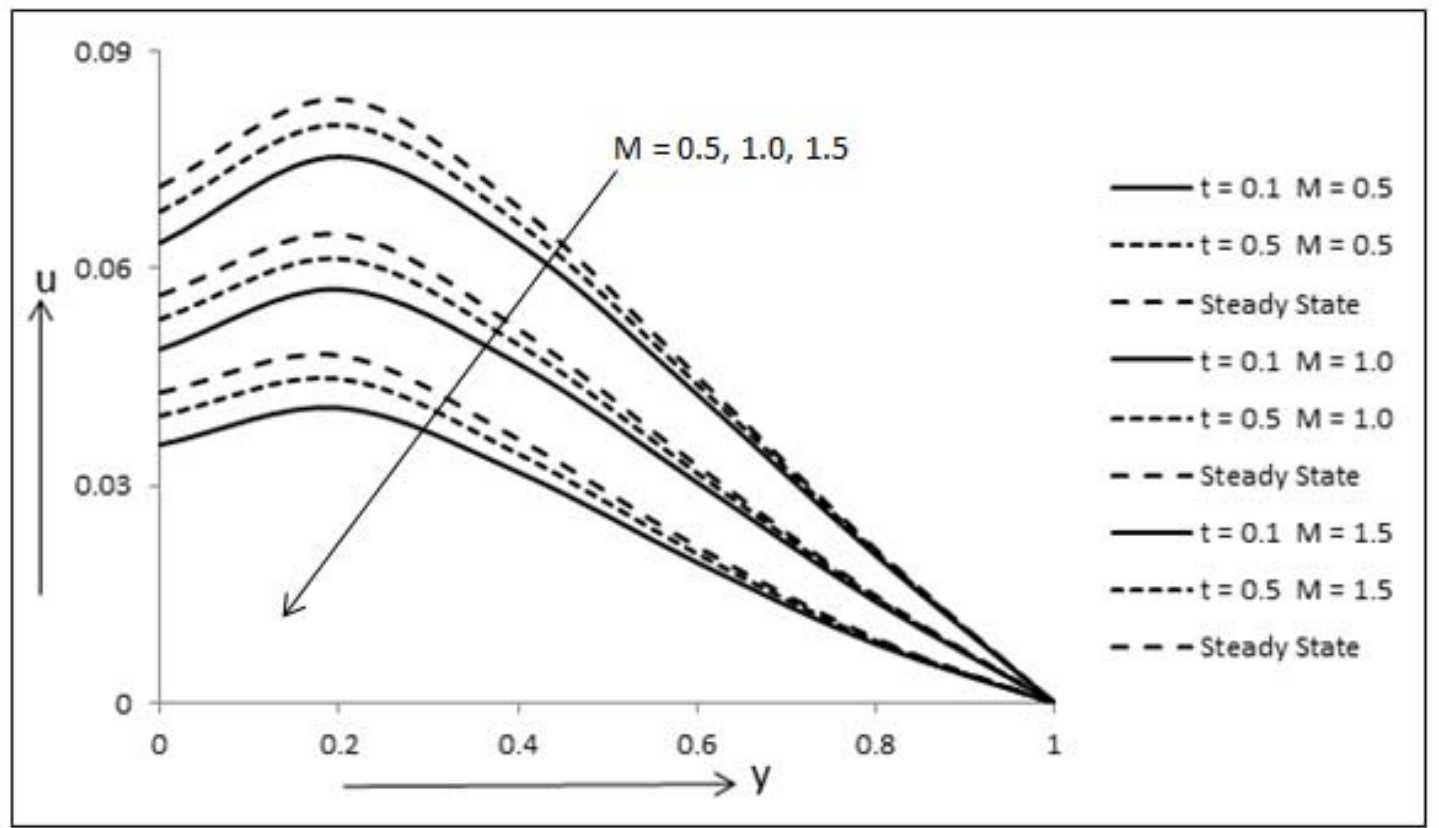

Fig.6. Velocity profiles for various values of the Hartmann number. 


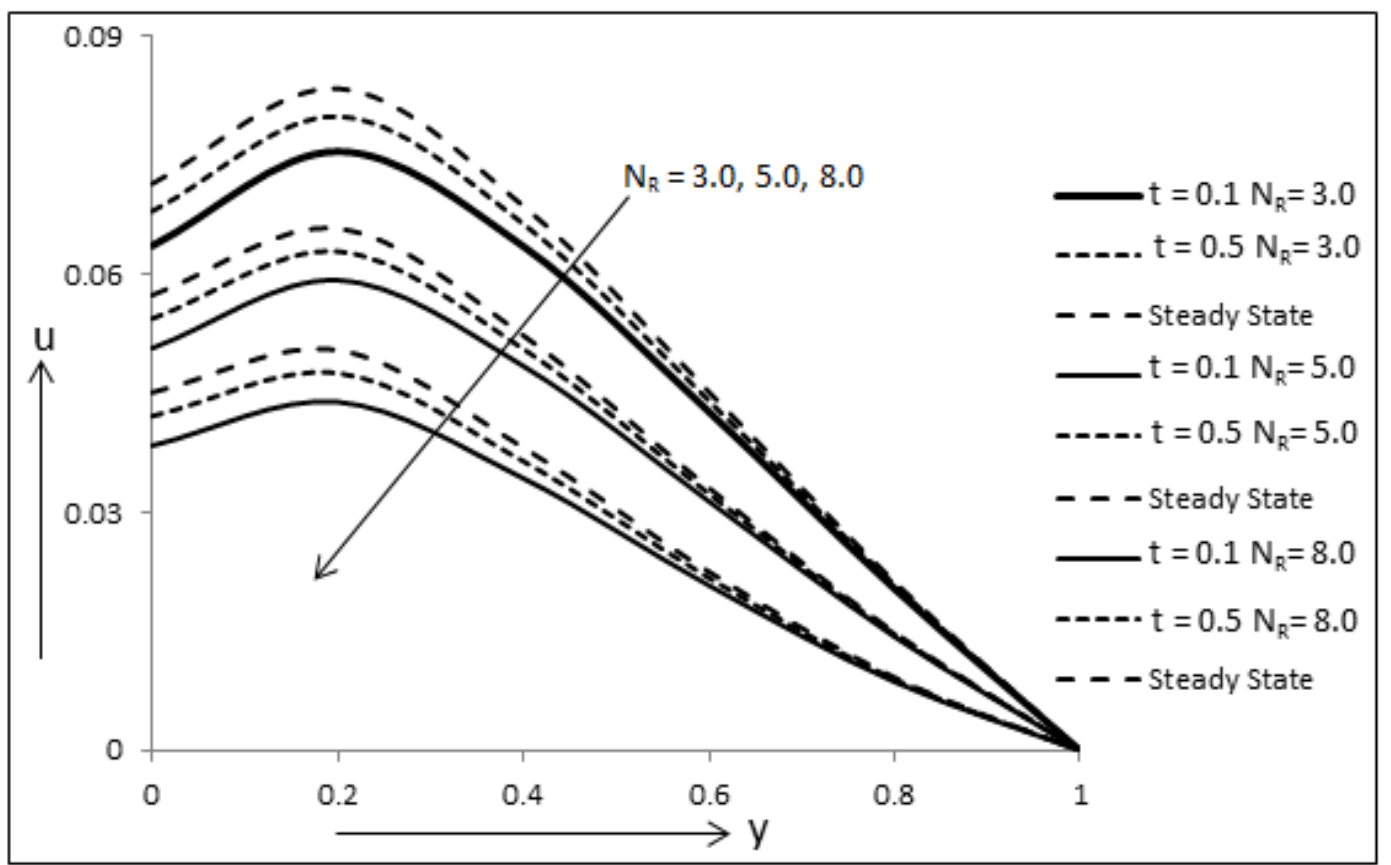

Fig.7. Velocity profiles for various values of the radiation parameter.

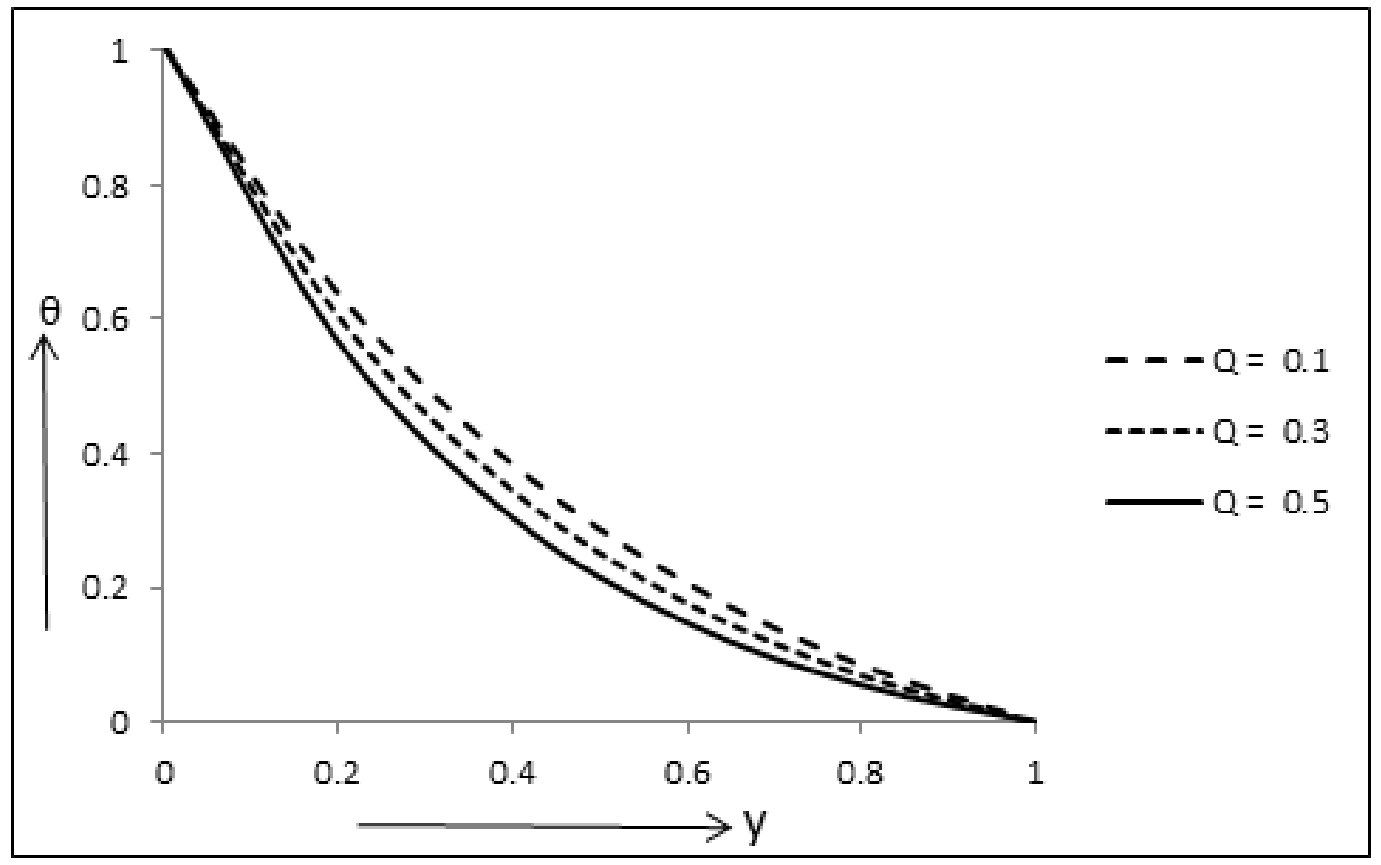

Fig.8. Temperature profiles for various values of the heat source parameter $N$. 


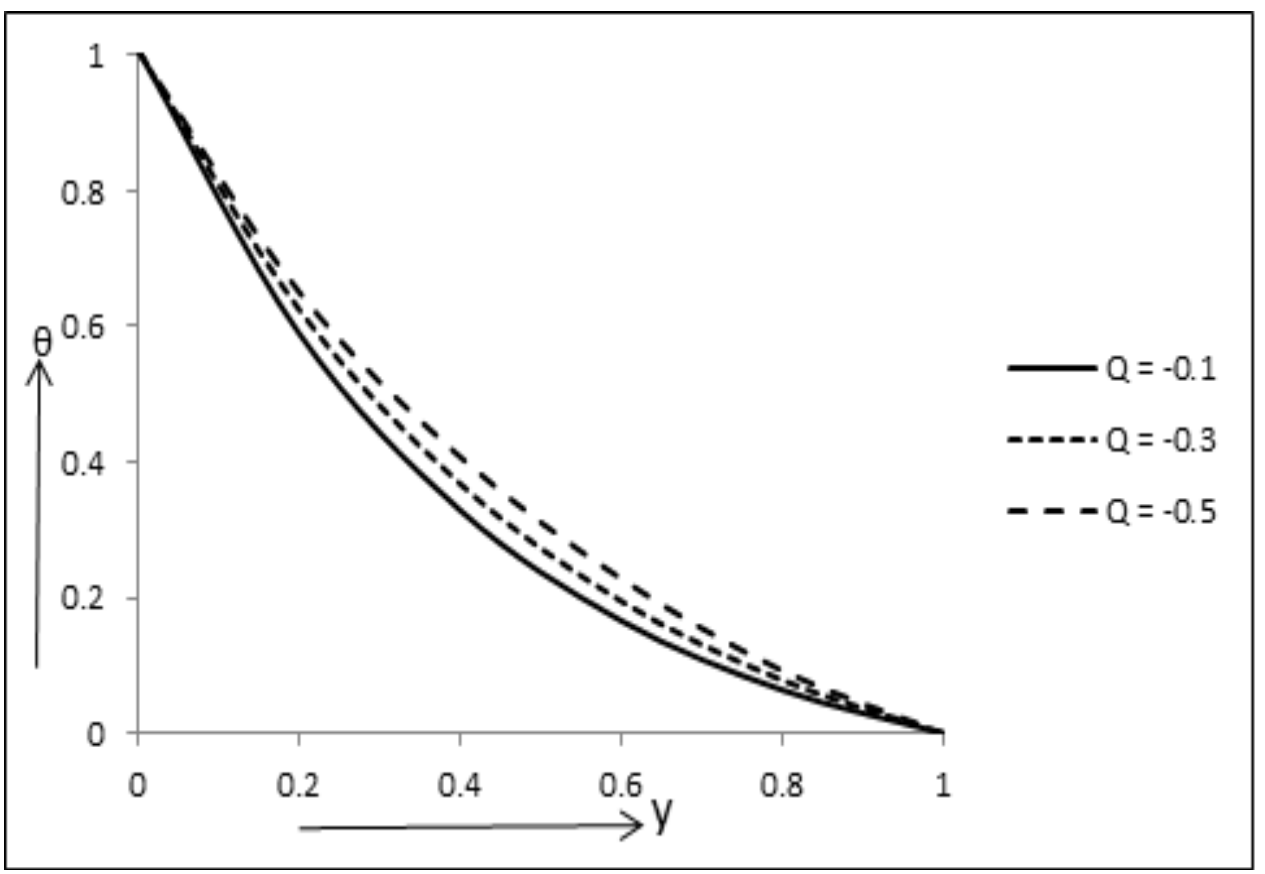

Fig.9. Temperature profiles for various values of the heat sink parameter.

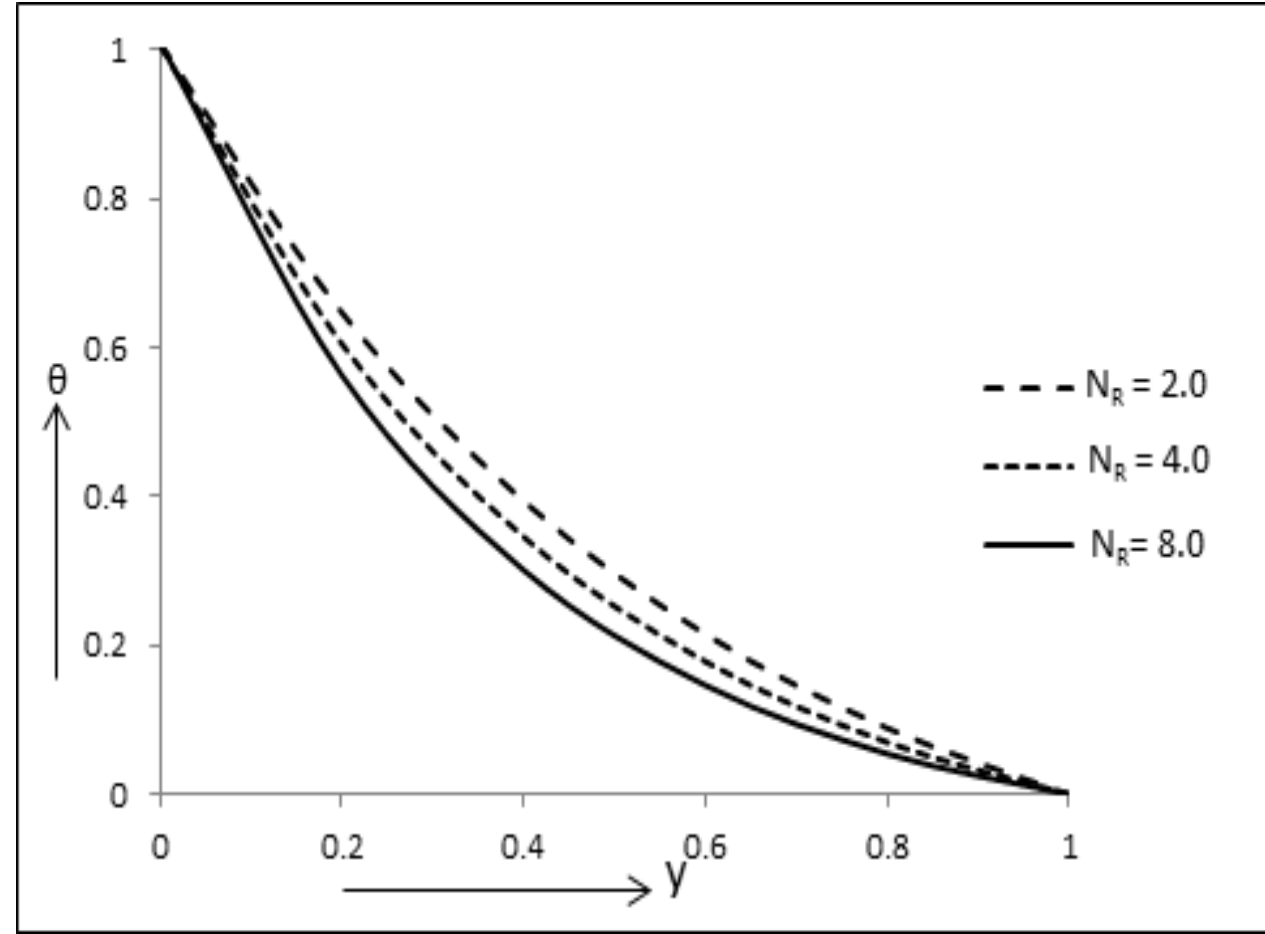

Fig.10. Temperature profiles for various values of the radiation parameter. 


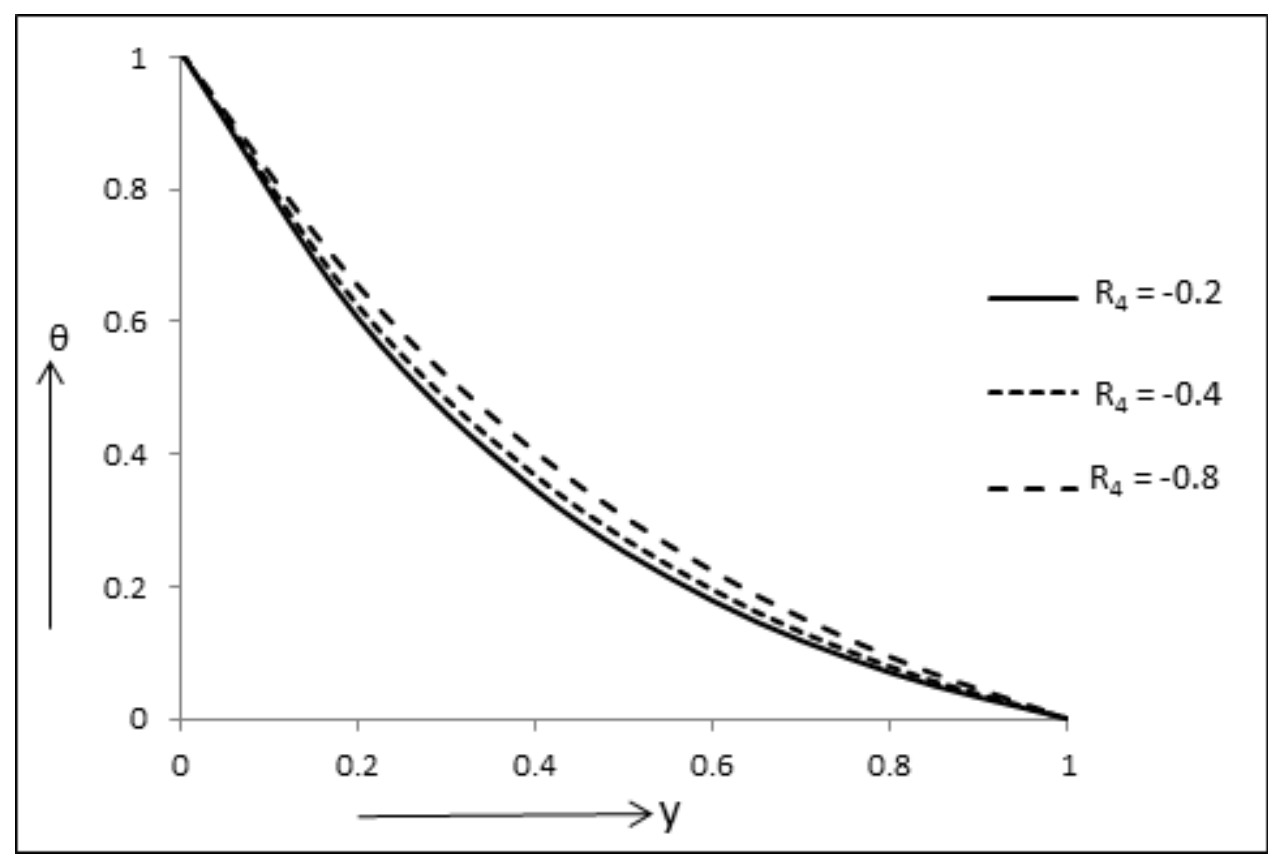

Fig.11. Temperature profiles for various values of the buoyancy force parameter $\left(R_{4}<0\right)$.

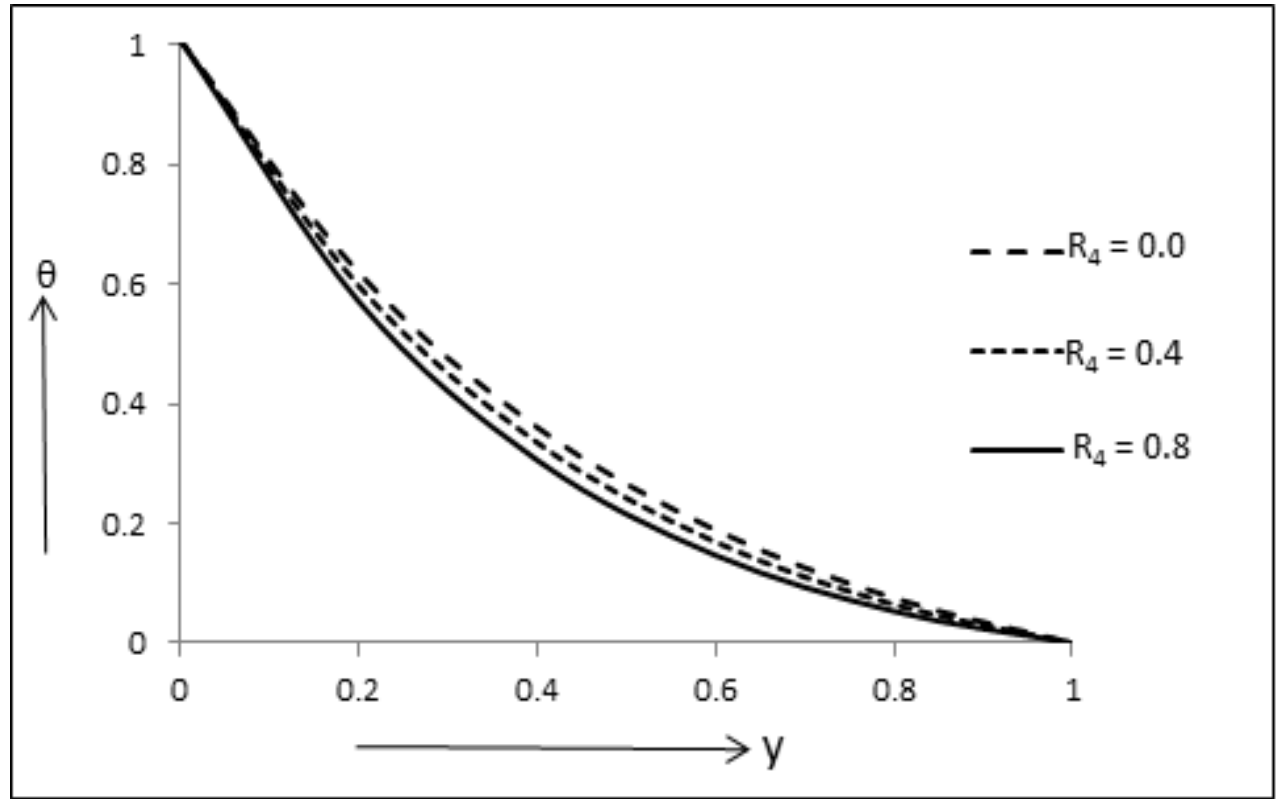

Fig.12. Temperature profiles for various values of the buoyancy force parameter $\left(R_{4}>0\right)$. 


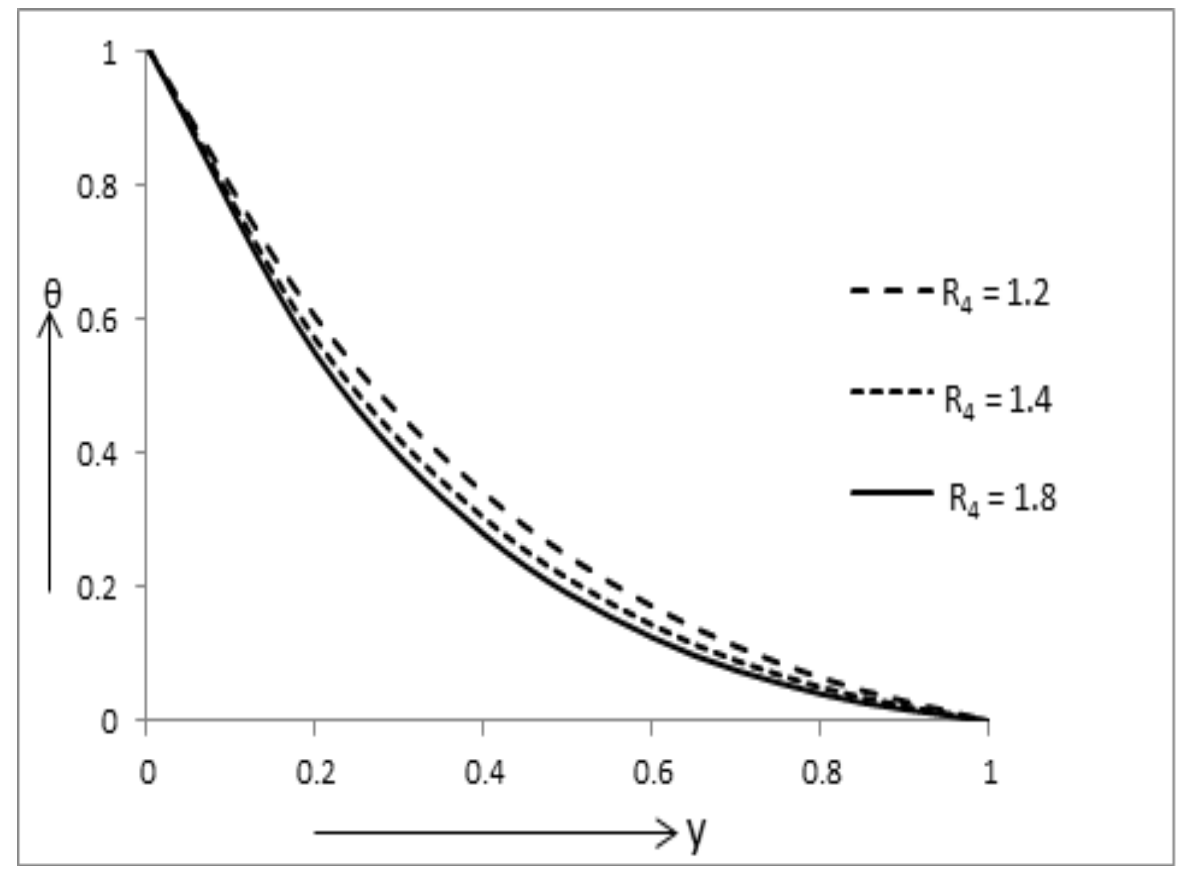

Fig.13. Temperature profiles for various values of the buoyancy force parameter $\left(R_{4}>1\right)$.

\section{Nomenclature}

$B_{0} \quad$ - uniform magnetic field

$C_{P}$ - specific heat at constant pressure

Da - Darcy number

F - Forchheimer constant

Gr - Grashof number

$g$ - acceleration due to gravity

$d$ - distance between vertical walls

$K$ - permeability of the porous medium

$k$ - thermal conductivity

M - Hartmann number

$n$ - non-dimensional positive constant

$n^{*}-$ small positive constant

Pr - Prandtl number

$Q$ - non-dimensional constant heat source

$Q_{0}$ - dimensional constant heat source

$R_{4}$ - buoyancy force distribution parameter

$T^{*}$ - temperature of the fluid

$T_{m}{ }^{*}$ - initial temperature of the fluid

$T_{h}{ }^{*} \quad$ - temperature of the heated wall

$T_{c}^{*} \quad$ - temperature of the cooled wall

$t$ - time in non-dimensional form

$t^{*} \quad$ time

$u^{*}$ - velocity of the fluid

$u$ - fluid velocity in non-dimensional form 


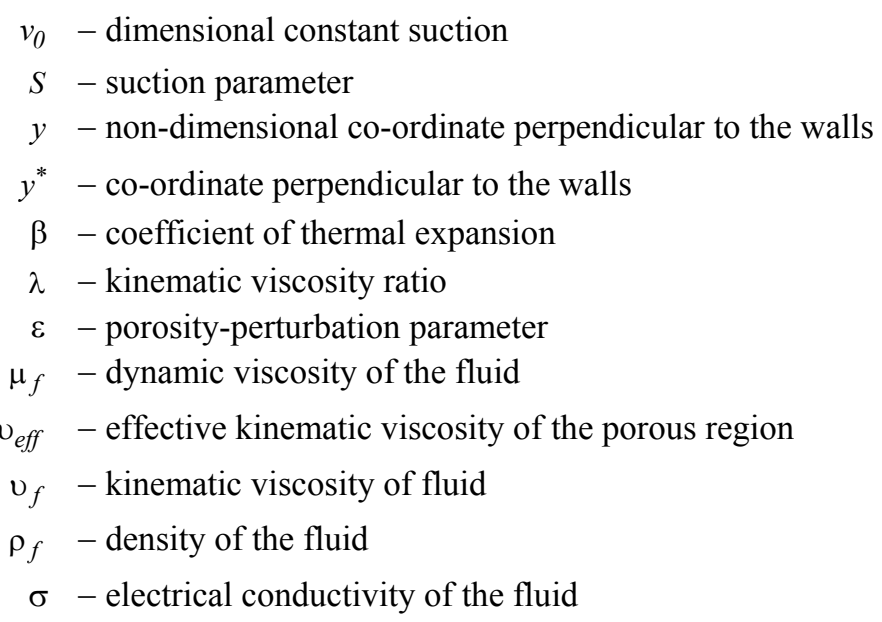

\section{Appendix}

$$
\begin{aligned}
& E_{1}=\frac{1}{\mathrm{D} \mathrm{a}}+\mathrm{M}, \quad E_{2}=\frac{1}{\mathrm{D} \mathrm{a}}+\mathrm{M}-n, \\
& n_{1}=\frac{-\operatorname{Pr} S+\sqrt{\operatorname{Pr}^{2} S^{2}+4 k_{2} \operatorname{Pr} Q}}{2 k_{2}}, \quad n_{2}=\frac{-\operatorname{Pr} S-\sqrt{\operatorname{Pr}^{2} S^{2}+4 k_{2} \operatorname{Pr} Q}}{2 k_{2}}, \\
& n_{4}=\frac{-\operatorname{Pr} S-\sqrt{\operatorname{Pr}^{2} S^{2}+4 k_{\backslash 2} \operatorname{Pr}(Q-n)}}{2}, \quad B_{1}=\frac{\operatorname{Pr} S n_{1} F_{1}}{k_{2} n_{1}{ }^{2}+\operatorname{Pr} S k_{2}-\operatorname{Pr}(Q-n)}, \\
& B_{2}=\frac{\operatorname{Pr} S n_{2} F_{2}}{k_{2} n_{2}{ }^{2}+\operatorname{Pr} S n_{2}-\operatorname{Pr}(Q-n)}, \quad B_{3}=e^{n_{3}}\left(1+B_{1}+B_{2}\right), \\
& B_{4}=B_{1} e^{n_{1}}+B_{2} e^{n_{2}}, \quad B_{5}=e^{n_{3}}-e^{n_{4}}, \quad B_{6}=e^{n_{4}}\left(1+B_{1}+B_{2}\right), \\
& B_{7}=B_{1} e^{n_{1}}+B_{2} e^{n_{2}}, \quad B_{8}=e^{n_{4}}-e^{n_{3}} \\
& F_{1}=\frac{e^{n_{2}}\left(R_{4}-1\right)-R_{4}}{e^{n_{1}}-e^{n_{2}}}, \quad F_{2}=\frac{R_{4}-e^{n_{1}}\left(R_{4}-1\right)}{e^{n_{1}}-e^{n_{2}}} \\
& F_{3}=\frac{B_{6}-B_{7}}{B_{8}}, \quad F_{4}=\frac{B_{3}-B_{4}}{B_{5}} \\
& n_{5}=\frac{-S+\sqrt{S^{2}+4 E_{1} \lambda}}{2 \lambda}, \quad n_{6}=\frac{-S-\sqrt{S^{2}+4 E_{1} \lambda}}{2 \lambda}
\end{aligned}
$$




$$
\begin{aligned}
& B_{9}=\frac{F_{1}}{\lambda n_{1}^{2}+S n_{1}-E_{1}} \\
& B_{10}=\frac{F_{2}}{\lambda n_{2}^{2}+S n_{2}-E_{1}} \\
& B_{11}=B_{9}\left[e^{n_{6}} n_{35}-e^{n_{1}} n_{15}\right], \quad B_{12}=B_{10}\left[e^{n_{6}} n_{36}-e^{n_{2}} n_{15}\right], \\
& B_{13}=\left[e^{n_{6}} n_{16}-e^{n_{5}} n_{15}\right], \quad B_{14}=\frac{\mathrm{Gr}}{\sqrt{\mathrm{Da}}}, \\
& B_{15}=\frac{B_{14} F_{5}{ }^{2}}{4 n_{5}{ }^{2} \lambda+2 S n_{5}-E_{1}}, \quad B_{16}=\frac{B_{14} F_{6}{ }^{2}}{4 n_{6}{ }^{2} \lambda+2 S n_{6}-E_{1}}, \\
& F_{5}=\frac{B_{11}+B_{12}}{B_{13}}, \quad F_{6}=\frac{B_{13}+B_{14}}{B_{15}}, \\
& B_{17}=\frac{B_{14} B_{9}{ }^{2}}{4 n_{1}{ }^{2} \lambda+2 S n_{1}-E_{1}}, \quad B_{18}=\frac{B_{14} B_{10}{ }^{2}}{4 n_{2}{ }^{2} \lambda+2 S n_{2}-E_{1}} \\
& B_{19}=\frac{2 F_{5} F_{6} B_{14}}{n_{9}{ }^{2} \lambda+S n_{9}-E_{1}}, \quad B_{20}=\frac{2 F_{5} B_{9} B_{14}}{n_{10}{ }^{2} \lambda+S n_{10}-E_{1}}, \\
& B_{21}=\frac{2 F_{5} B_{10} B_{14}}{n_{11}{ }^{2} \lambda+S n_{11}-E_{1}}, \quad B_{22}=\frac{2 F_{6} B_{9} B_{14}}{n_{12}{ }^{2} \lambda+S n_{12}-E_{1}} \\
& B_{23}=\frac{2 F_{6} B_{10} B_{14}}{n_{13}{ }^{2} \lambda+S n_{13}-E_{1}}, \quad B_{24}=\frac{2 B_{9} B_{10} B_{14}}{n_{14}{ }^{2} \lambda+S n_{14}-E_{1}}, \\
& B_{25}=B_{15}+B_{16}+B_{17}+B_{18}+B_{19}-B_{20}-B_{21}-B_{22}-B_{23}+B_{24}, \\
& B_{26}=B_{15} 2 n_{5}+B_{16} 2 n_{6}+B_{17} 2 n_{1}+B_{18} 2 n_{2}+B_{19} n_{9}+ \\
& -B_{20} n_{10}-B_{21} n_{11}-B_{22} n_{12}-B_{23} n_{13}+B_{24} n_{14} \text {, } \\
& B_{27}=B_{15} e^{2 n_{5}}+B_{16} e^{2 n_{6}}+B_{17} e^{2 n_{1}}+B_{18} e^{2 n_{2}}+B_{19} e^{n_{9}}+ \\
& -B_{20} e^{n_{10}}-B_{21} e^{n_{11}}-B_{22} e^{n_{12}}-B_{23} e^{n_{13}}+B_{24} e^{n_{14}}, \\
& F_{7}=\frac{e^{n_{6}} h B_{26}-e^{n_{6}} B_{25}+B_{27} n_{15}}{e^{n_{6}} n_{16}-e^{n_{5}} n_{15}}, \quad F_{8}=\frac{e^{n_{5}} h B_{26}-e^{n_{5}} B_{25}+B_{27} n_{16}}{e^{n_{5}} n_{15}-e^{n_{6}} n_{16}}, \\
& n_{7}=\frac{-S+\sqrt{S^{2}+4 E_{2} \lambda}}{2 \lambda}, \quad n_{8}=\frac{-S-\sqrt{S^{2}+4 E_{2} \lambda}}{2 \lambda},
\end{aligned}
$$




$$
\begin{aligned}
& n_{9}=n_{5}+n_{6}, \quad n_{10}=n_{1}+n_{5}, \quad n_{11}=n_{2}+n_{5}, \quad n_{12}=n_{1}+n_{6}, \\
& n_{13}=n_{2}+n_{6}, \quad n_{14}=n_{1}+n_{2}, \quad n_{15}=1-h n_{6}, \quad n_{16}=1-h n_{5}, \\
& n_{17}=1-h n_{8}, \quad n_{18}=1-h n_{7}, \quad n_{19}=n_{5}+n_{7}, \quad n_{20}=n_{5}+n_{8}, \\
& n_{21}=n_{3}+n_{5}, \quad n_{22}=n_{4}+n_{5}, \quad n_{23}=n_{6}+n_{7}, \quad n_{24}=n_{6}+n_{8}, \\
& n_{25}=n_{3}+n_{6}, \quad n_{26}=n_{4}+n_{6}, \quad n_{27}=n_{1}+n_{7}, \quad n_{28}=n_{1}+n_{8}, \\
& n_{29}=n_{1}+n_{3}, \quad n_{30}=n_{1}+n_{4}, \quad n_{31}=n_{2}+n_{7}, \quad n_{32}=n_{2}+n_{8}, \\
& n_{33}=n_{2}+n_{3}, \quad n_{34}=n_{2}+n_{4}, \quad n_{35}=1-h n_{1}, \quad n_{36}=1-h n_{2}, \\
& B_{28}=\frac{B_{1}}{n_{1}{ }^{2} \lambda+S n_{1}-E_{2}}, \quad B_{29}=\frac{B_{2}}{n_{2}{ }^{2} \lambda+S n_{2}-E_{2}}, \quad B_{30}=\frac{F_{3}}{n_{3}{ }^{2} \lambda+S n_{3}-E_{2}}, \\
& B_{31}=\frac{F_{4}}{n_{4}{ }^{2} \lambda+S n_{4}-E_{2}}, \quad B_{32}=\frac{S B_{9}}{n_{1}{ }^{2} \lambda+S n_{1}-E_{2}}, \quad B_{33}=\frac{S B_{10}}{n_{2}{ }^{2} \lambda+S n_{2}-E_{2}}, \\
& B_{34}=\frac{S F_{5}}{n_{5}{ }^{2} \lambda+S n_{5}-E_{2}}, \quad B_{35}=\frac{S F_{6}}{n_{6}{ }^{2} \lambda+S n_{6}-E_{2}}, \\
& B_{36}=B_{28}+B_{29}-B_{30}-B_{31}+B_{32}+B_{33}-B_{34}-B_{35}, \\
& B_{37}=n_{1}\left(B_{28}+B_{32}\right)+n_{2}\left(B_{29}+B_{33}\right)-n_{3} B_{30}-n_{4} B_{31}-n_{5} B_{33}-n_{6} B_{35}, \\
& B_{38}=e^{n_{1}}\left(B_{28}+B_{32}\right)+e^{n_{2}}\left(B_{29}+B_{33}\right)-e^{n_{3}} B_{30}-e^{n_{4}} B_{31}-e^{n_{5}} B_{33}-e^{n_{6}} B_{35}, \\
& F_{9}=\frac{e^{n_{8}} h B_{37}-e^{n_{8}} B_{36}+B_{38} n_{17}}{e^{n_{8}} n_{18}-e^{n_{7}} n_{17}}, \quad \quad F_{10}=\frac{e^{n_{7}} h B_{37}-e^{n_{7}} B_{36}+B_{38} n_{18}}{e^{n_{7}} n_{17}-e^{n_{8}} n_{18}}, \\
& B_{39}=\frac{2 \mathrm{Gr}}{\sqrt{\mathrm{Da}}}, \quad B_{40}=F_{5} B_{34} B_{39}-S 2 n_{5} B_{15}, \quad B_{41}=F_{6} B_{35} B_{39}+S 2 n_{6} B_{16}, \\
& B_{42}=\left[B_{39} B_{10}\left(B_{33}+B_{29}\right)+2 n_{2} B_{18} S\right], \quad B_{43}=\left[B_{39} B_{9}\left(B_{28}+B_{32}\right)+2 n_{1} B_{17} S\right], \\
& B_{44}=\left[B_{39}\left(F_{5} B_{35}+F_{6} F_{34}\right)+B_{19} S\left(n_{5}+n_{6}\right)\right], \quad B_{45}=\left[B_{39}\left\{F_{5}\left(B_{28}+B_{32}\right)+B_{9} B_{34}\right\}+B_{20} S n_{10}\right], \\
& B_{46}=\left[B_{39}\left\{F_{5}\left(B_{29}+B_{33}\right)+B_{10} B_{34}\right\}+B_{21} S n_{11}\right], \quad B_{47}=\left[B_{39}\left\{F_{6}\left(B_{28}+B_{32}\right)+B_{9} B_{35}\right\}+B_{22} S n_{12}\right], \\
& B_{48}=\left[B_{39}\left\{F_{6}\left(B_{29}+B_{33}\right)+B_{9} B_{35}\right\}+B_{23} S n_{12}\right],
\end{aligned}
$$




$$
\begin{aligned}
& B_{49}=\left[B_{39}\left\{B_{10}\left(B_{28}+B_{32}\right)+B_{9}\left(B_{33}+B_{29}\right)\right\}+B_{24} S n_{13}\right], \quad B_{50}=B_{39} F_{5} F_{9}, \\
& B_{51}=B_{39} F_{5} F_{10}, \quad B_{52}=B_{39} F_{5} B_{33}, \quad B_{53}=B_{39} F_{5} B_{31}, \quad B_{54}=B_{39} F_{6} F_{9}, \\
& B_{55}=B_{39} F_{6} F_{10}, \quad B_{56}=B_{39} F_{6} B_{30}, \quad B_{57}=B_{39} F_{6} B_{31}, \quad B_{58}=B_{39} F_{9} B_{9}, \\
& B_{59}=B_{39} F_{10} B_{9}, \quad B_{60}=B_{39} B_{9} B_{30}, \quad B_{61}=B_{39} B_{9} B_{31}, \quad B_{62}=B_{39} F_{9} B_{10}, \\
& B_{63}=B_{39} F_{10} B_{10}, \quad B_{64}=B_{39} B_{10} B_{30}, \quad B_{65}=B_{39} B_{10} B_{31}, \quad B_{66}=S n_{5} F_{7}, \\
& B_{67}=S n_{6} F_{8}, \quad B_{68}=\frac{B_{40}}{\lambda 4 n_{5}{ }^{2}+2 n_{5} S-E_{2}}, \quad B_{69}=\frac{B_{41}}{\lambda 4 n_{6}{ }^{2}+2 n_{6} S-E_{2}}, \\
& B_{70}=\frac{B_{42}}{\lambda 4 n_{2}{ }^{2}+2 n_{2} S-E_{2}}, \quad B_{71}=\frac{B_{43}}{\lambda 4 n_{1}{ }^{2}+2 n_{1} S-E_{2}}, \quad B_{72}=\frac{B_{44}}{\lambda n_{9}{ }^{2}+S n_{9}-E_{2}}, \\
& B_{73}=\frac{B_{45}}{\lambda n_{10}{ }^{2}+S n_{10}-E_{2}}, \quad B_{74}=\frac{B_{46}}{\lambda n_{11}^{2}+S n_{11}-E_{2}}, \quad B_{75}=\frac{B_{47}}{\lambda n_{12}^{2}+S n_{12}-E_{2}}, \\
& B_{76}=\frac{B_{48}}{\lambda n_{13}{ }^{2}+S n_{13}-E_{2}}, \quad B_{77}=\frac{B_{49}}{\lambda n_{14}{ }^{2}+S n_{14}-E_{2}}, \quad B_{78}=\frac{B_{50}}{\lambda n_{19}{ }^{2}+S n_{19}-E_{2}}, \\
& B_{79}=\frac{B_{51}}{\lambda n_{20}{ }^{2}+S n_{20}-E_{2}}, \quad B_{80}=\frac{B_{52}}{\lambda n_{21}^{2}+S n_{21}-E_{2}}, \quad B_{81}=\frac{B_{53}}{\lambda n_{22}{ }^{2}+S n_{22}-E_{2}}, \\
& B_{82}=\frac{B_{54}}{\lambda n_{23}{ }^{2}+S n_{23}-E_{2}}, \quad B_{83}=\frac{B_{55}}{\lambda n_{24}{ }^{2}+S n_{24}-E_{2}}, \quad B_{84}=\frac{B_{56}}{\lambda n_{25}^{2}+S n_{25}-E_{2}}, \\
& B_{85}=\frac{B_{57}}{\lambda n_{26}{ }^{2}+S n_{26}-E_{2}}, \quad B_{86}=\frac{B_{58}}{\lambda n_{27}^{2}+S n_{27}-E_{2}}, \quad B_{87}=\frac{B_{59}}{\lambda n_{28}^{2}+S n_{28}-E_{2}}, \\
& B_{88}=\frac{B_{60}}{\lambda n_{29}{ }^{2}+S n_{29}-E_{2}}, \quad B_{89}=\frac{B_{61}}{\lambda n_{30}{ }^{2}+S n_{30}-E_{2}}, \quad B_{90}=\frac{B_{62}}{\lambda n_{31}{ }^{2}+S n_{31}-E_{2}}, \\
& B_{91}=\frac{B_{63}}{\lambda n_{32}^{2}+S n_{32}-E_{2}}, \quad B_{92}=\frac{B_{64}}{\lambda n_{33}^{2}+S n_{33}-E_{2}}, \quad B_{93}=\frac{B_{65}}{\lambda n_{34}^{2}+S n_{34}-E_{2}}, \\
& B_{94}=\frac{B_{66}}{\lambda n_{5}^{2}+S n_{5}-E_{2}}, \quad B_{95}=\frac{B_{67}}{\lambda n_{6}^{2}+S n_{6}-E_{2}},
\end{aligned}
$$




$$
\begin{aligned}
& B_{96}=-B_{68}-B_{69}-B_{70}-B_{71}-B_{72}+B_{73}+B_{74}+B_{75}+B_{76}-B_{77}+B_{78}+B_{79}+ \\
& -B_{80}-B_{81}+B_{82}+B_{83}-B_{84}-B_{85}-B_{86}-B_{87}+B_{88}+B_{89}-B_{90}-B_{91}+ \\
& +B_{92}+B_{93}-B_{94}-B_{95}, \\
& F_{11}=\frac{e^{n_{8}} B_{97}-e^{n_{8}} B_{96}+n_{17} B_{98}}{\left[e^{n_{8}} n_{18}-e^{n_{7}} n_{17}\right]}, \quad F_{12}=\frac{e^{n_{7}} B_{97}-e^{n_{7}} B_{96}+n_{18} B_{98}}{\left[e^{n_{7}} h_{17}-e^{n_{8}} n_{18}\right]}, \\
& B_{97}=\left[-2 n_{5} B_{68}-2 n_{6} B_{69}-2 n_{2} B_{70}-2 n_{1} B_{71}-B_{72} n_{9}+\right. \\
& +B_{73} n_{10}+B_{74} n_{11}+B_{75} n_{12}+B_{76} n_{13}-B_{77} n_{14}+B_{78} n_{19}+ \\
& +B_{79} n_{20}-B_{80} n_{21}-B_{81} n_{22}+B_{82} n_{23}+B_{83} n_{24}-B_{84} n_{25}-B_{85} n_{26}-B_{86} n_{27}+ \\
& \left.-B_{87} n_{28}+B_{88} n_{29}+B_{89} n_{30}-B_{90} n_{31}-B_{91} n_{32}+B_{92} n_{33}+B_{93} n_{34}-n_{5} B_{94}-n_{6} B_{95}\right] h, \\
& B_{98}=-e^{2 n_{5}} B_{68}-e^{2 n_{6}} B_{69}-e^{2 n_{2}} B_{70}-e^{2 n_{1}} B_{71}-B_{72} e^{n_{9}}+B_{73} e^{n_{10}}+B_{74} e^{n_{11}}+B_{75} e^{n_{12}}+B_{76} e^{n_{13}}+ \\
& -B_{77} e^{n_{14}}+B_{78} e^{n_{19}}+B_{79} e^{n_{20}}-B_{80} e^{n_{21}}-B_{81} e^{n_{22}}+B_{82} e^{n_{23}}+B_{83} e^{n_{24}}-B_{84} e^{n_{25}}-B_{85} e^{n_{26}}-B_{86} e^{n_{27}}+ \\
& -B_{87} e^{n_{28}}+B_{88} e^{n_{29}}+B_{89} e^{n_{30}}-B_{90} e^{n_{31}}-B_{91} e^{n_{32}}+B_{92} e^{n_{33}}+B_{93} e^{n_{34}}-B_{94} e^{n_{5}}-B_{95} e^{n_{6}} .
\end{aligned}
$$

\section{References}

[1] Kaviany M. (1995): Principles of Heat Transfer in Porous media'. - 2, New York: Springer.

[2] Pop I. and Ingham D.B. (2001): Convective Heat Transfer: Mathematical and computational modeling of viscous fluids and porous Pergamon. Oxford.

[3] Ingham D.B. and Pop I. (2005): Transport Phenomena in Porous Media. - 3, United Kingdom: Elsevier.

[4] Vadasz P. (2008): Emerging Topics in Heat and Mass Transfer in Porous Media. - New-York: Springer.

[5] Vafai K. (2010): Porous Media: Application in Biological Systems and Biotechnology. - CRC Press.

[6] Nield A.N. and Bejan A. (2013): Mechanics of fluid flow through a porous medium. - Springer.

[7] Irmay S. (1958): On the theoretical derivation of Darcy and Forchheimer formulas. - EOS, Trans. AGU., vol.39, pp.702-707.

[8] Neale G. and Nader W. (1974): Practical significance of Brinkman's extension of Darcy law: Couple parallel flows with in a channel and bounding a porous channel. - Canadian J. Chem. Eng., vol.52, pp.475-478.

[9] Kavinay M. (1985): Laminar flow, through a porous channel by isothermal parallel plates. - Int. J. Heat Mass Transfer, vol.28, pp.851-858.

[10] Nakayama A., Kayama H. and Kuwahara F. (1988): Analysis on forced convection in a channel filled with a Brinkman-Darcy-porous medium, exact and approximate solutions. - Vol.23, pp.291-95.

[11] Cheng P.A. and Choudhary A. (1988): Forced convection in the entrance region of a packed channel with asymmetric heating. - ASME J. Heat Transfer, vol.110, pp.946-54.

[12] Vafai K. and Kim S.J. (1989): Forced convection in a channel filled with a porous medium: an exact solution. ASME J. Heat Transfer, vol.111, pp.1103-1106.

[13] Nakayama A., Kokudai T. and Koyama H. (1990): Forchheimer free convection over a non-isothermal of arbitrary shape in a saturated porous medium. - ASME J. Heat Transfer, vol.112, pp.511-15.

[14] Kladias N. and Prasad V. (1991): Experimental verification of Darcy-Brinkman-Forchheimer-model for natural convection in porous media. - AIAA J. Thermodyphys Heat Transfer, vol.5, pp.560-75. 
[15] Shenoy A.V. (1993): Darcy-Forchheimer-natural forced and mixed convection heat transfer in non-Newtonian power law fluid-saturated porous medium. - Transp Porous Med., vol.11, No.3, pp.219-241.

[16] Vafai K. and Kim S.J. (1995): On the limitations of the Brinkman-Forchheimer Darcy equation. - Int. J. Heat Fluid Flow, vol.16, pp.11-15.

[17] Whitakar S. (1996): The Forchheimer equation: a theoretical development. - Transport Porous Med., vol.25, pp.27-61.

[18] Nield D.A., Junqueira S.L.M. and Lage J.L. (1996): Forced convection in a fluid saturated Porous medium channel with isothermal or isoflux boundaries. - J. Fluid Mech., vol.322, pp.201-214.

[19] Kuznetsov A.V. and Austria V. (1998): Analytical investigation of heat transfer in Coutte flow through a Porous medium utilizing the Brinkman-Forchheimer-extended Darcy model. - Acta Mechanica, vol.129, pp.13-24.

[20] Nakayama A. (1998): A unified treatment of Darcy-Forchheimer boundary layer flows. - Transport Phenomena in Porous Media, vol.1, pp.179-204.

[21] Leong K.C. and Jin L.W. (2004): Heat transfer of oscillating and steady flows in a channel filled with porousmedium. - Int. Commun. Heat Mass Transfer, vol.31, pp.63-72.

[22] Singh A.K. and Takhar H.S. (2005): Free convection flow of two immiscible viscous liquids through parallel permeable beds: use of Brinkman model. - Int. J. Fluid Mech. Res., vol.32, pp.635-650.

[23] Singh A.K., Kumar R., Singh U., Singh N.P. and Singh A.K. (2011): Unsteady hydromagnetic convective flow in a vertical channel using Darcy-Brinkman-Forchheimer-extended-model with heat generation/absorption: Analysis with asymmetric heating/cooling of the channel walls. - Int. J. of Heat and Mass Transfer, vol.54, pp.5633-5642.

[24] Pal, Dulal and Mondal, Hiranmoy (2012): Hydromagnetic convective diffusion of species in Darcy-Forchheimer porous medium with non-uniform heat source/sink and variable viscosity. - Int. Commu. Heat and Mass Transfer, vol.39, pp.913-917.

[25] Rapits A., Massalas C. and Tzivanids G. (1982): Hydromagnetic free convection flow through porous medium between two parallel plates. - Phys. Lett., vol.90, pp.288-289.

[26] Attia H.A. and Kotb N.A. (1996): MHD flow between two parallel plates with heat transfer. - Acta Mechanica, vol.117, pp.215-220.

[27] Kim Y.I. (2000): Unsteady MHD convective heat transfer past semi-infinite vertical porous moving plate with variable suction. - Int. J. Engineering Science, vol.38, pp.833-845.

[28] Attia H.A. (2006): On the effectiveness of variation in the physical variables on MHD steady flow between parallel plates with heat transfer. - Int. J. for Numerical Method in Engineering, vol.65, pp.224-35.

[29] Ahmed A. (2007): Effects of unsteady free convective MHD flow through a porous medium bounded by an infinite vertical porous plate. - Bull. Cal. Math. Soc., vol.99, pp.511-22.

[30] Das U.N., Deka R.K. and Soundalgekar V.M. (1996): Radiation effects on flow past an impulsively started vertical infinite plate. - J. Theo. Mech., vol.1, pp.111-115.

[31] Bakier A.Y. (2001): Thermal radiation effects on mixed convection from vertical surfaces in saturated porous media. - Int. Comm. Heat and Mass Transfer, vol.28, pp.243-248.

[32] Sanyal D.C. and Adhikari A. (2006): Effects of radiation on MHD vertical channel flow. - Int. J. App. Mech. and Engg., vol.4, pp.817- 821.

[33] Mebine P. (2007): Radiation effects on MHD Couette flow with heat transfer between two parallel plates. Global Journal of Pure and Applied Mathematics, vol.3, pp.191-202.

[34] El-Hakim M.A. and Rashad A.M. (2007): Effect of radiation on non-Darcy free convection from a vertical cylinder embedded in a fluid-saturated porous medium with a temperature-dependent viscosity. - J. Porous Media, vol.10, pp.209-218. 
[35] Muthucumarasamy R. and Kulandivel T. (2008): Radiation effects on moving vertical plate with variable temperature and uniform mass diffusion. - Energy Heat and Mass Transfer, vol.30, pp.79-88.

[36] Singh K.D. and Kumar R. (2009): Radiation effects on the exact solution of free convective oscillatory flow through porous medium in a rotating vertical porous channel. - Rajasthan Acad. Physical Science, vol.8, pp.295-310.

[37] Singh K.D. and Garg B.P. (2010): Exact solution of an oscillatory free convection MHD flow on a rotating porous channel with radiative heat. - Proc. Nat. Acad. Sci., India Sect., vol.80, pp.81-89.

[38] Khandelwal K., Gupta A., Poonam and Jain N.C. (2003): Effects of couple stresses on the flow through a porous medium with variable permeability in slip flow regime. - J. Ganita, vol.54, No.2, pp.203-12.

[39] Sharma P.K. and Choudhary R.C. (2003): Effects of variable suction on transient free convective viscous incompressible flow past a vertical plate with periodic temperature variation in slip flow regime. - Emirates Journal of Engineering Research, vol.8, No.2, pp.33-38.

[40] Sharma P.K. (2005): Influence of periodic temperature and concentration on unsteady free convective viscous incompressible flow and heat transfer past a vertical plate in slip flow regime. - J. Mathematics, vol.13, No.1, pp.51-62.

[41] Chaudhary R.C and Jha A.K. (2008): Effects of chemical reactions on MHD micropolar fluid flow past a vertical plate in slip flow regime. - J. of Applied Mathematics and Mechanics, vol.29, No.9, pp.1179-1194.

[42] Singh K.D. and Pathak R. (2013): Effects of slip conditions and hall current on an oscillatory convective MHD flow in a rotating vertical porous channel with thermal radiation. - International Journal of Applied Mathematics and Mechanics, vol.9, No.12, pp.60-77.

[43] Alazmi B. and Vafai K. (2000): Analysis of variants with the porous media transport models. - ASME J. Heat Transfer, vol.122, pp.303-326.

[44] Ergun S. (1952): Fluid flow through packed columns. - Chemical Engineering Progress, vol.48, pp.89-94.

[45] Gebhart B. and Pera L. (1971): The natural of vertical convection flows resulting from the combined buoyancy effects of thermal and mass diffusion. - Int. J. Heat and Mass Transfer, vol.14, pp.2025-2050.

[46] Chamkha A.J. (2002): On laminar hydromagnetic mixed convection in a vertical channel with symmetric and asymmetric wall heating conditions. - Int. J. Heat Mass Transfer, vol.45, pp.2509-2525.

[47] Paul T., Singh A.K. and Mishra A.K. (2006): Transient free convection flow in a porous region bounded by two vertical walls heated / cooled asymmetrically. - J. Energy Heat Mass Transfer, vol.28, pp.193-207.

Received: May 6, 2017

Revised: March 16, 2018 